\title{
The time gap between repeated re-happening opportunistic infections among people living with HIVIAIDS commencing antiretroviral treatment
}

\author{
Habtamu Mellie Bizuayehu \\ Debre Markos University, College of medicine and health science, public health department, Debre Markos, Ethiopia
}

Email address:

habtamumellie@yahoo.com

To cite this article:

Habtamu Mellie Bizuayehu. The Time Gap between Repeated Re-Happening Opportunistic Infections among People Living with HIV/AIDS Commencing Antiretroviral Treatment. Clinical Medicine Research. Vol. 4, No. 1, 2015, pp. 11-16. doi: 10.11648/j.cmr.20150401.13

\begin{abstract}
Introduction: According to 2011 Ethiopian demographic health survey, the national and Amhara Regional state administration adult HIV prevalence was $1.5 \%$ and $2.2 \%$ respectively. The major causes of morbidity and mortality of PLWHA (people living with HIV/AIDS) patients are OIs (opportunistic infections) that would occur in up to $40 \%$ of PLWHA. OIs are repeatedly happening in HIV (Human Immunodeficiency Virus) infected patients though there is no prior local evidence on time gap of repetition. Therefore the current study is aimed to determine time gap between repeated re-happening OIs and its associated factors among PLWHA who are initiated ART (Anti-Retroviral Treatment). Method: Institution based retrospective cohort study was conducted among 364 systematically selected PLWHA commencing ART. Time-gap was estimated using Kaplan-meier survival and actuarial life table. Hazard rate was calculated using Cox proportional-hazard model. Result: during follow up OIs were re-diagnosed in about three quarter $(76.9 \%)$ of participants. In each week the probability of getting the re-happened OI was 1.1 per 100 persons. The median duration of staying free of OI re-happening was 66 weeks. In multivariate analysis using logistic regression, educational status, marital status, Prophylaxisis exposure, ART and Prophylaxisis drug adherence, hemoglobin and CD4 level were significantly associated with time gap of relapse. Thus organizations working on HIV/AIDS should further work to enhance time gap of relapse.
\end{abstract}

Keywords: HIV/AIDS, Re-Happening, ART, PLWHA, Ethiopia

\section{Introduction}

Globally about 34 million people were living with HIV in $2012[1,2]$. Still, there were about 2.2 million new infections [3]. Since the beginning of the epidemic nearly 30 million people have died of AIDS-related causes [1, 2, 4].

At end of 2010 about 22.9 million which is $67 \%$ of those living with HIV/AIDS globally are in Africa though only about $12 \%$ of the world's population lives in the region [2]. In terms of mortality, the region represents about $79 \%$ of AIDS mortality globally [5], the estimated mortality from AIDS related illnesses at end of 2010 are 1.2 million [2].

According to 2011 Ethiopian demographic health survey, HIV prevalence in Ethiopia was $1.5 \%$ and in the study area of Amhara region, it was $2.2 \%$ [6].

In Ethiopia the fee based and universal free access Antiretroviral (ARV) treatment was started in 2002/3 and 2004/5 respectively. The country uses decentralizing the ARV treatment service provision to the level of Health centers and private Health facilities for fast expansion of the service [7].

The major causes of morbidity and mortality of HIV/AIDS patients are OIs [8] that would occur in up to $40 \%$ of PLWHA with a CD4 count less than 250 cells $/ \mathrm{mm} 3$ [9].

In North India, TB was the commonest OI (71\%) followed by candidiasis $(39.3 \%), P C P(7.4 \%)$, cryptococcal meningitis and cerebral toxoplasmosis (3.7\% each) [10].

A national study in Ethiopia showed HIV patients' had OIs like Herpes Zoster scar (19.3\%); pulmonary tuberculosis $(5.2 \%)$ and pneumonia $(5.2 \%)$ and some patients $(2 \%)$ had more than one neurologic complications of HIV/AIDS [11]. In Northwest Ethiopia about $7.5 \%$ and $8.3 \%$ of the HIV patients' had pulmonary tuberculosis and cryptococcal meningitis respectively $[12,13]$. Nearly a quarter $(22.7 \%)$ of HIV patients' had chronic diarrhea in Southern Ethiopia [14].

Even though, OIs are prevalent in the study area there is no local evidence on time gap between repeated re-happening OIs after prior treatment among PLWHA who are initiated ART. Thus the current study would give OI free time and its 
associated factors that can be used to plan resources and to identify PLWHA who need especial care. The evidence is expected to be used by governmental and non-governmental organizations working on HIV/AIDS.

\section{Methods and Materials}

\subsection{Study Design, Area and Population}

Institution based retrospective cohort study was conducted from January 1, 2008 up to December 31, 2013 in Debre Markos town governmental health institutions among 4412 PLWHA who are initiated ART. In the town, there is one referral hospital and one health center that provide chronic HIV care. All 18 years old and above PLWHA who develop OI after 30 days of starting ART (the first 30 days after HAART were excluded due to most immune reconstitution inflammatory syndrome occurring in the period [15]) and taking standard treatment according to the Ethiopian Ministry of Health guideline were the study populations. HIV patients who take treatment for OI but not returned at least once to health institution for follow up; those who did not develop any OI since registered on HIV care after starting ART; and their follow up format incompletely documented when treatment for OI given or on consecutive follow ups were excluded from the study.

\subsection{Sampling and Data Collection Procedure}

The sample size was calculated based on the assumption of $95 \%$ confidence interval and $2.5 \%$ of absolute precision and the proportion of pulmonary tuberculosis $(6 \%)$ among PLWHA who are initiating ART [16]. The calculated sample size using OpenEpi Version 2.3, May 2009 was 347 and after adding 5\% contingency the final sample size was becoming 364 .

After preparing the sampling frame among PLWHA commencing ART that fulfill the inclusion criteria, selection of study participants were done using simple random sampling technique via random number table method.

Data collection instrument was developed from both federal ministry of health chronic HIV care form and the patient's card in which the follow up health status data were registered. Then the needed data was collected by reviewing ART follow up form, laboratory request and patients' card. If laboratory examinations like CD4 count, Hemoglobin, weight are not found during entry and exit to the study, the measurements that are most nearest to time of entry and exit to study were taken as baseline and end line predictors respectively.

Participants whose future time re-happening of OI not confirmed due to loss follow-up/drop-out/transferred out/dead by any disease other than OI/cause of death not confirmed during study period or not develop OI at end of the study period were censored.

Health professionals working on ART clinics were collecting the data after taking appropriate training on objective of the study and about the data collection instrument in detail.

\subsection{Operational Definitions}

Re-happening/relapse/re-diagnosis: diagnosis of OI by health personnel working in ART clinic after completing the preceding OI treatment

Survival: OI free duration or not re-happening of OI

Censored: None re-happening of OI in study participant during follow up on study; but future re-happening is not certain.

Drop out: if PLWHA on HIV care lost to follow-up above 3 months as recorded by health personnel working on ART clinic.

Lost to follow-up: if PLWHA on HIV care not seen for $>1$ month as recorded by health personnel working on ART clinic.

Transferred-out: if PLWHA on HIV care in one health institution shift to other health institution.

Good Adherence: if PLWHA adherent $>95 \%$ that is the percentage of missed dose is $<2$ dose of 30 doses or $<3$ dose of 60 dose as documented by health personnel working on ART clinic.

Fair Adherence: if PLWHA adherent 85-94\% that is the percentage of missed dose is 3-5 dose of 30 doses or 3-9 dose of 60 doses as documented by health personnel working on ART clinic.

Poor Adherence: if PLHIV adherent $<85 \%$ that is the percentage of missed dose is $>6$ doses of 30 doses or $>9$ dose of 60 doses as documented by health personnel working on ART clinic.

\subsection{Statistical Analysis}

A coded questionnaire was double entered in to Epi Info version 3.5.1 statistical package by a trained data clerk and exported to SPSS version 20 and STATA version 11 statistical packages for analysis of statistical inferences. Before further analysis, data cleaning was done using frequencies, cross tabulations, sorting and listing to check missed values and outliers. Errors identified during the process were corrected by revising the original questionnaire.

To estimate the time of OI free duration, the actuarial life table and Kaplan Meier survival was used. Assumption of proportional-hazard was checked using Schoenfeld residual with $p$-value $>0.1(\alpha=10 \%)$ and the assumption was not violated. Base line and end line hemoglobin value was correlated $(r=0.48, p=0.006)$ thus end line hemoglobin value was excluded from multivariate analysis due to affecting the final model by its redundancy nature which affects precision of estimate. The hazard rate at uni-variate and multivariate level was calculated using Cox proportional-hazard model. Variables having $p$-value $<0.05$ at uni-variate analysis and not collinear was entered into final model of multivariate analysis to identify associated factors with outcome.

\subsection{Ethical Consideration}

Ethical approval and clearance was given by the School of Public Health Addis Ababa University ethical committee. Permission was also obtained from the concerned bodies of 
East Gojam zone and Debre Markos town Health Department and the responsible bodies of hospital and health centers. To protect confidentiality, health professionals working in ART clinics prepared the sampling frame and extracted the data from medical records. In addition no personal identifier was extracted on medical records.

\section{Result}

In the six year follow up period majority of the participants were females (64.6\%), orthodox Christians (91.6\%), living in urban $(74.5 \%)$, married $(46.4 \%)$, not educated $(41.5 \%)$ and not employed $(74.2 \%)$ in governmental or private sectors. Their median age was 32 years in which all most all of them were below 50 years old (table 1).

The base line and end line CD4 count values were 159 and 313 cells/ul respectively. The respective base line and end line mean values for hemoglobin were $11.9( \pm 2.5)$ and 12.4 $( \pm 1.9) \mathrm{g} / \mathrm{dl}$ and for body mass index it was $18.9( \pm 3)$ and 19.7 $( \pm 2.9) \mathrm{kg} / \mathrm{m}^{2}$. At start of the study about $72 \%$ of the participants were diagnosed only one type of OI while the rest was diagnosed 2 or more OIs at one visit of health institution. Of the diagnosed OIs at start, about half (51.1\%) was having WHO stage III OI. All most all (98.4\%) the participants have no other concomitant chronic diseases like hypertension, cardiac disease, and diabetes mellitus. Nearly all study subjects were having working functional status both at base line (71.7\%) and at follow up (89\%). All participants were on first line ART regimens in which about $40.4 \%$ and $56.9 \%$ were taking Tenofovirdisoproxilfumarate + Lamivudine + Efavirenz regimen both at base line and at end line respectively and their drug adherence status was good both at base line (94.8\%) and at follow up (93.7\%). Most of the study subjects were taking Prophylaxisis both at base line (93.1\%) and at follow up (92.3\%) and their drug adherence status was good both at base line $(95.7 \%)$ and at follow up (94.5\%) (Table1).

\subsection{Time Gap of OI Re-Happening and Associated Factors}

During follow up the cumulative incidence of OI rehappening was $76.9 \%(95 \% \mathrm{CI}$ : 72.6-81.25) and the incidence rate was 1.1 (95\% CI: $0.97-1.23)$ per 100 person weeks. The commonly re-happening OIs were recurrent upper respiratory tract infection $(19.3 \%)$, bacterial pneumonia $(12.1 \%)$, oral candidiasis $(10.4 \%)$, chronic diarrhea $(9.3 \%)$, herpes zoster $(9.3 \%)$, pulmonary tuberculosis $(6.1 \%)$, extra pulmonary tuberculosis $(7.1 \%)$, PCP (3.9\%), encephalopathy (3.9\%), toxoplasmosis $(3.2 \%)$, and other types (3.3\%).

According to Kaplan Meier survival estimation, the median time of OI re-happening was 66 (95\% CI: $57.87-$ 74.13) weeks (figure 1). As the actuarial life table analysis showed the probability of free of OI re-happening with in the first five weeks was $97 \%$ and it was becoming $<10 \%$ and $<1 \%$ after 180 and 255 weeks respectively.

After adjustment for potential confounders in multivariate cox proportional hazard model, the factors that delay rehappening of OIs were being educated than non-educated, taking Prophylaxisis at follow up, having a hemoglobin level above $10 \mathrm{~g} / \mathrm{dl}$ at base line, having a CD4 level above 100 compared $<100$ cells/ul both at base line and at end line. But being widowed compared to married and not adhering ART drug at base line were risks for short time re-happening of OIs (Table 1).

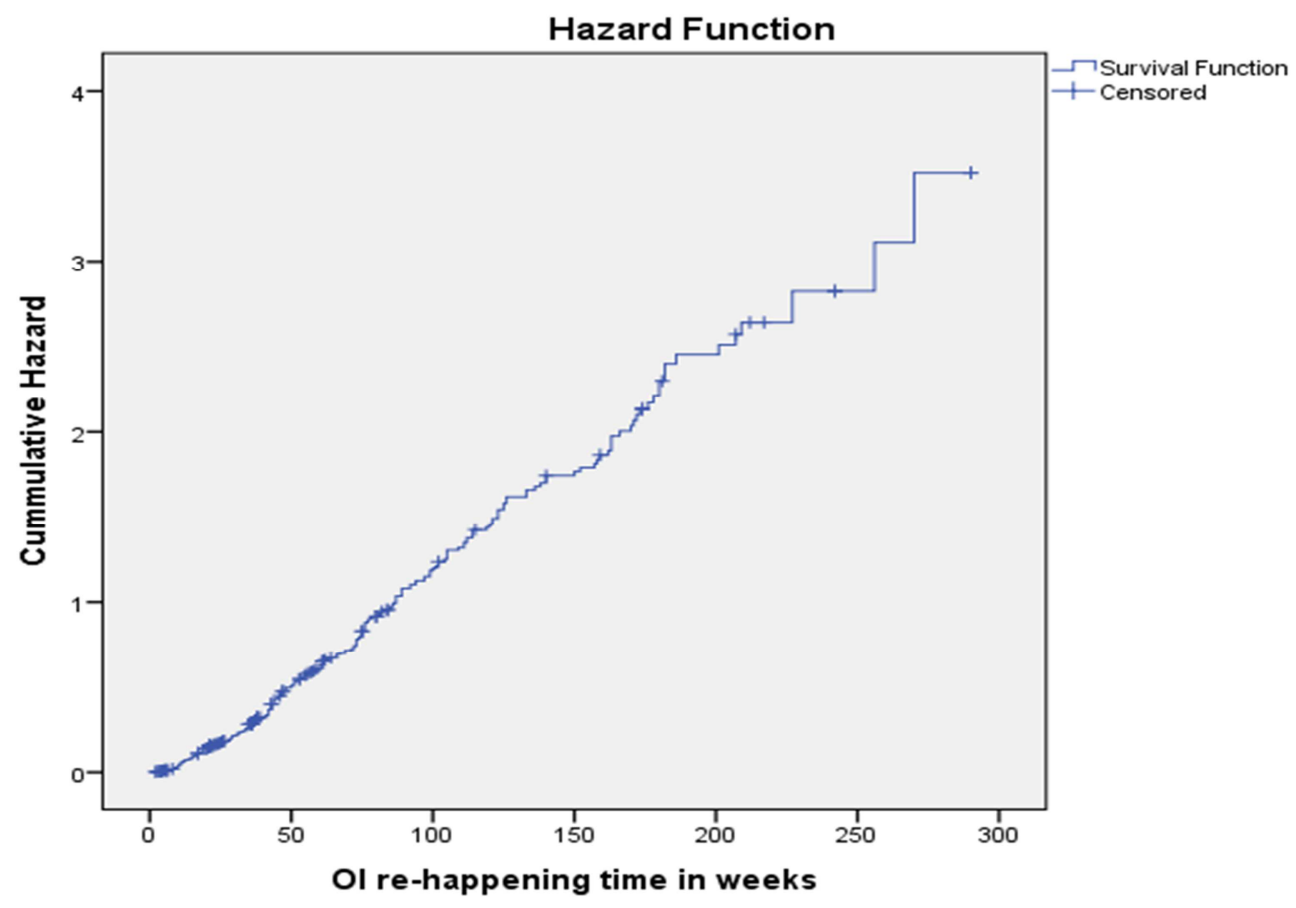

Figure 1. kaplan-Meier survival estimation of through time diagnosis of OI re-happening among ART PLWHA in Debre Markos town between 2008 and 2013. 
Table 1. The cumulative incidence ofre-happened OIs, Kaplan Meier survival estimation of median duration of not diagnosing re-happened OI and cox proportional hazard model of the association between factors and OI re-happening among ART PLWHA in Debre Markos town between 2008 and 2013.

\begin{tabular}{|c|c|c|c|c|c|c|}
\hline \multirow{2}{*}{ Variables } & & \multicolumn{2}{|c|}{ Diagnosis of Re-happening OI } & \multirow{2}{*}{ Median KMS } & \multirow{2}{*}{ CHR (95\% CI) } & \multirow{2}{*}{$\operatorname{AHR}(95 \% \mathrm{CI})$} \\
\hline & & No (\%) & Yes $(\%)$ & & & \\
\hline \multirow{4}{*}{ Marital status } & Married & $45(53.6)$ & $124(44.3)$ & 73 & 1 & 1 \\
\hline & Single & $13(15.5)$ & $48(17.1)$ & 79 & $1.15(0.82-1.60)$ & $0.71(0.42-1.21)$ \\
\hline & Divorced & $18(21.4)$ & $76(27.1)$ & 72 & $1.11(0.83-1.49)$ & $0.81(0.52-1.27)$ \\
\hline & Widowed & $8(9.5)$ & $32(11.4)$ & 35 & $2.12(1.43-3.14)$ & $4.65(2.13-10.16)$ \\
\hline \multirow{4}{*}{ Educational status } & Not educated & $21(25)$ & $130(46.4)$ & 61 & 1 & 1 \\
\hline & Grade 1-8 & $19(22.6)$ & $82(29.3)$ & 54 & $0.76(0.57-1.0)$ & $0.78(0.47-1.28)$ \\
\hline & Grade 9-12 & $21(25)$ & $53(18.9)$ & 75 & $0.62(0.45-0.87)$ & $0.25(0.13-0.48)$ \\
\hline & Above grade 12 & $23(27.4)$ & $15(5.4)$ & 170 & $0.38(0.22-0.64)$ & $0.14(0.05-0.43)$ \\
\hline \multirow{2}{*}{ Occupational status } & Un-employed & $47(56)$ & $223(79.6)$ & 56 & 1 & 1 \\
\hline & Employed & $37(44)$ & $57(20.4)$ & 92 & $0.61(0.46-0.82)$ & $0.85(0.51-1.41)$ \\
\hline \multirow{4}{*}{ WHO staging ${ }^{\mathrm{B}}$. } & I & $3(3.6)$ & $22(7.9)$ & 72 & 1 & 1 \\
\hline & II & $30(35.7)$ & $77(27.5)$ & 85 & $0.49(0.31-0.79)$ & $0.80(0.35-1.80)$ \\
\hline & III & $42(50)$ & $144(51.4)$ & 61 & $0.53(0.33-0.83)$ & $0.52(0.23-1.16)$ \\
\hline & IV & $9(10.7)$ & $37(13.2)$ & 47 & $0.61(0.36-1.04)$ & $0.32(0.098-1.01)$ \\
\hline \multirow{2}{*}{ Prophylaxisis exposure ${ }^{\mathrm{B} .}$} & No & $4(4.8)$ & $21(7.5)$ & 33 & 1 & 1 \\
\hline & Yes & $80(95.2)$ & $259(92.5)$ & 72 & $0.48(0.31-0.75)$ & $0.24(0.08-0.69)$ \\
\hline \multirow{3}{*}{ Prophylaxisis exposure ${ }^{\mathrm{F} .}$} & No & $5(6)$ & $23(8.2)$ & 21 & 1 & 1 \\
\hline & Yes & 79(94) & 257(91.8) & 69 & $0.61(0.40-0.94)$ & $0.41(0.16-1.03)$ \\
\hline & Good & $84(100)$ & 247(94.3) & 72 & 1 & 1 \\
\hline \multirow[t]{3}{*}{ Prophylaxisis adherence ${ }^{B}$} & Fair & $0(0)$ & $6(2.3)$ & 67 & $1.0(0.45-2.26)$ & $0.21(0.05-1.0)$ \\
\hline & Poor & $0(0)$ & $9(3.4)$ & 35 & $3.37(1.71-6.64)$ & $3.62(0.32-41)$ \\
\hline & Good & $82(97.6)$ & $244(93.5)$ & 72 & 1 & 1 \\
\hline \multirow{2}{*}{ Prophylaxisis adherence F } & Fair & $2(2.4)$ & $6(2.3)$ & 45 & $2.30(1.01-5.23)$ & $3.44(0.91-12.97)$ \\
\hline & Poor & $0(0)$ & $11(4.2)$ & 35 & $3.42(1.85-6.32)$ & $4.48(0.98-20.50)$ \\
\hline \multirow{3}{*}{ ART adherence ${ }^{\mathrm{B} .}$} & Good & $80(95.2)$ & $265(94.6)$ & 71 & 1 & 1 \\
\hline & Fair & $4(4.8)$ & $7(2.5)$ & 42 & $1.71(0.81-3.64)$ & $15.35(3.12-75.55)$ \\
\hline & Poor & $0(0)$ & $8(2.9)$ & 24 & $4.18(2.05-8.53)$ & $4.21(0.39-45.83)$ \\
\hline \multirow{3}{*}{ ART adherence ${ }^{\mathrm{F}}$} & Good & $83(98.8)$ & $258(92.1)$ & 72 & 1 & 1 \\
\hline & Fair & $1(1.2)$ & $9(3.2)$ & 41 & $2.32(1.19-4.53)$ & $0.97(0.34-2.65)$ \\
\hline & Poor & $0(0)$ & $13(4.6)$ & 24 & $3.69(2.1-6.50)$ & $1.04(0.16-6.80)$ \\
\hline \multirow{5}{*}{ CD4 count (cells $/ \mu \mathrm{l})^{\mathrm{B} .}$} & $<=100$ & $9(10.7)$ & $23(8.2)$ & 66 & 1 & 1 \\
\hline & 101-199 & $19(22.6)$ & $49(17.5)$ & 75 & $0.60(0.36-0.99)$ & $0.29(0.12-0.71)$ \\
\hline & $200-350$ & $25(29.8)$ & $98(35)$ & 61 & $0.76(0.48-1.20)$ & $0.60(0.26-1.38)$ \\
\hline & $351-499$ & $18(21.4)$ & $70(25)$ & 50 & $0.69(0.43-1.12)$ & $1.30(0.52-3.24)$ \\
\hline & $>=500$ & $13(15.5)$ & $40(14.3)$ & 123 & $0.33(0.19-0.56)$ & $0.87(0.34-2.26)$ \\
\hline \multirow{5}{*}{ CD4 count $(\text { cells } / \mu 1)^{\mathrm{F}}$} & $<=100$ & $4(10.3)$ & $14(7.4)$ & 45 & 1 & 1 \\
\hline & 101-199 & $6(15.4)$ & $26(13.8)$ & 75 & $0.57(0.29-1.09)$ & $1.19(0.45-3.15)$ \\
\hline & $200-350$ & $12(30.8)$ & $68(36.2)$ & 83 & $0.50(0.28-0.90)$ & $0.87(0.36-2.10)$ \\
\hline & $351-499$ & $11(28.2)$ & $51(27.1)$ & 101 & $0.30(0.16-0.57)$ & $0.31(0.12-0.78)$ \\
\hline & $>=500$ & $6(15.4)$ & $29(15.4)$ & 121 & $0.24(0.12-0.46)$ & $0.24(0.10-0.60)$ \\
\hline \multirow{2}{*}{ Hemoglobin value $(\mathrm{g} / \mathrm{dl})^{\mathrm{B}}$. } & $<10$ & $17(31.5)$ & $96(39)$ & 44 & 1 & 1 \\
\hline & $>=10$ & $37(68.5)$ & $150(61)$ & 74 & $0.60(0.46-0.78)$ & $0.58(0.39-0.88)$ \\
\hline \multirow{2}{*}{$\begin{array}{l}\text { Extra pulmonary } \\
\text { uberculosis }{ }^{\mathrm{B}}\end{array}$} & No & $77(91.7)$ & $267(95.4)$ & 66 & 1 & 1 \\
\hline & Yes & $7(8.3)$ & $13(4.6)$ & 72 & $0.43(0.26-0.73)$ & $3.26(0.94-11.28)$ \\
\hline \multirow{2}{*}{ Oral candidiasis ${ }^{\mathrm{B} .}$} & No & $74(88.1)$ & $249(88.9)$ & 69 & 1 & 1 \\
\hline & Yes & 10(11.9) & $31(11.1)$ & 66 & $1.83(1.24-2.72)$ & $1.08(0.57-2.03)$ \\
\hline \multirow{2}{*}{ Pneumonia ${ }^{\text {B. }}$} & No & $78(92.9)$ & $237(84.6)$ & 72 & 1 & 1 \\
\hline & Yes & $6(7.1)$ & $43(15.4)$ & 45 & $1.44(1.04-2.0)$ & $2.73(1.38-5.42)$ \\
\hline
\end{tabular}

Key,

B. $=$ base line value

F. $=$ follow up value

$\mathrm{CI}=$ confidence interval

KMS=Kaplan Meier survival in weeks

$\mathrm{CHR}=$ crude hazard rate

$\mathrm{AHR}=$ Adjusted hazard rate

\section{Discussion}

In current study, the cumulative incidence of OI rehappening was $76.9 \%$ and the commonly re-happening OIs were recurrent upper respiratory tract infection (19.3\%), bacterial pneumonia (12.1\%), oral candidiasis (10.4\%), chronic diarrhea $(9.3 \%)$, and herpes zoster $(9.3 \%)$. And this finding was nearly in agreement with prior studies $[9,10,12$ $14,16]$ though some figures are slightly vary among prior findings each other and with the current study due to differences in study design (prior ones are cross sectionals), 
and study area which is conducted in various socio-economic characteristics.

With regarding sex, various studies having contradicting outcome as risk for OI. In a cohort study, female sex increases the risk of toxoplasmic encephalitis [17]. A cohort study in United states showed female gender, were associated with significantly higher odds of OIs like herpes simplex virus-2 infection [18]. In contrary, a study in Thailand showed male gender was significantly associated with higher incidence of OIs after commencing ART [19]. In current study sex is not significantly associated and the possible reasons might be vary in study population, study design and differences in sociocultural contexts of the source population.

One of the factor that delay OI re-happening in current study was having a CD4 count above 100 compared $<100$ cells/ul both at base line and at end line and this finding was in conformity with other studies [17, 20-23]. The HIV cohort study in Switzerland showed the baseline CD4 count is one of the predictor for OI progression [20]. Another cohort study also showed higher CD4 cell count was associated with a reduction of risk of new OI progression [23].

The current finding shown as exposure for prophylaxis at follow up would delay repeated re-happening of OIs and this is in supported by other studies [21, 24-27]. Primary prophylaxis with Trimethoprim-sulfamethoxazole is preventing OIs [24]. Cotrimoxazole prophylaxis prevents diarrhea among PLWHA after ART initiation [26].

In current study not adhering ART drug at base line was the risk for short time re-happening of OIs and the result was supported by two studies $[12,28]$ in which non-adherence of ART was the risk for failure of the drug which enhances OI spread.

\section{Conclusion and Recommendation}

OIs were re-diagnosed in majority of participants. In each week, re-happened OIs would be seen in one out of hundred PLWHA. The median duration of staying free of OI rehappening was 66 weeks. Factors preventing/delaying short time re-happening of OI were being educated, taking Prophylaxisis, having a hemoglobin level above $10 \mathrm{~g} / \mathrm{dl}$, and having a CD4 level above 100 cells/ul. But being widow compared to married and not adhering ART were risks for frequent visiting of heath institutions due to re-happened OI illness. Thus health professionals working on ART clinic or organizations working on HIV/AIDS at local, regional or national level should further work to enhance duration between repeated OI re-diagnosis. Giving further counseling on ART drug adherence and taking of prophylaxis is vital to delay frequent visiting of health institutions due to rehappened OI illness. Boosting the CD4 count and hemoglobin value by drug or nutritional treatment or by other means is a key to prevent short duration OI re-happening. Social agencies working on marriage should further encourage and counsel PLWHA to marry in order to reduce illnesses by frequent relapsed OIs.

\section{Acknowledgment}

First of all, my deepest gratitude goes to Professor Getnet Mitkie, Dr Alemayehu Worku and Dr Fikre Enquselassie for their continuous and unreserved supports on completion of the work. In addition I would like to thank data collectors and supervisors and the managers of health institutions for giving permission to conduct the study.

\section{References}

[1] U.S. global health policy fact sheet, the global HIV/AIDS epidemic November 2011.

[2] Global HIV/AIDS response - epidemic update and health sector progress towards universal access - Progress report 2011.

[3] Together we will end AIDS. Geneva, Joint United Nations Programme on HIV/AIDS. 2012.

[4] UNAIDS World AIDS day report, regional fact sheet 2012.

[5] Edward J.M., Jean B.N., Iain B., James O., Amir A. et al. Adherence to antiretroviral therapy in Sub-Saharan Africa and North America: A meta-analysis. JAMA. 2006; 296(6):679-690.

[6] Central Statistical Agency (CSA), Ethiopia demographic and health survey 2011; final draft report, Addis Ababa Ethiopia, ICF International Calverton, Maryland, USA. March 2012.

[7] Annual performance report of multi-sectoralHIV/ AIDS response, federal HIV/AIDS prevention and control office 2002 E.C. $(2009 / 2010)$.

[8] Mariam Z.T., Abebe G., Mulu A.Opportunistic and other intestinal parasitic infections in AIDS patients, HIV seropositive healthy carriers and HIV sero-negative individuals in southwest Ethiopia. Dec 2008; 5(3):169-73.

[9] Gallant J.M., Chaisson R. Prophylaxis for opportunistic infections in patients with HIV infection. Ann Intern Med 1994; 120:932-44.

[10] SK Sharma, Tamilarasu K., Amit B., Tarun G.,Indrish B., et al Spectrum of clinical disease in a series of 135 hospitalized HIV-infected patients from north India. BMC Infectious Diseases 22 November 2004; 4:52.

[11] Tesfaye B., Yilma M. and Amanuel A. The pattern and predictors of mortality of HIV/AIDS patients with neurologic manifestation inEthiopia: a retrospective study. AIDS Research and Therapy 2012; 9:11

[12] Yitayih W., Dagnachew M. and Yeshambel B. Prevalence of Pulmonary tuberculosis and immunological profile of HIV coinfected patients in Northwest Ethiopia. BMC Research Notes 2012; 5:331.

[13] Seboxa T., Alemu S., Assefa A., Asefa A., Diro E. Cryptococcal meningitis in patients with acquired immunudeficiency syndrome in prehaart era at Gondar College of Medical Sciences Hospital north-west Ethiopia. Ethiop Med J. 2010 Jul;48(3):237-41.

[14] Shimelis A., Berhanu E., Girmay M., Zelalem A. and Techalew $\mathrm{S}$. Intestinal parasitic infections in relation to HIV/AIDS status, diarrhea and CD4 T-cell count. BMC Infectious Diseases 2009; 9:155 
[15] Surendra K.S., Sahajal D., Parag B.,Tamilarasu K., Sanjay R.,et al. a study of $\mathrm{TB}$ associated immune reconstitution inflammatory syndrome using the consensus case definition. Indian J Med Res 131, June 2010, pp 804-808

[16] N. Kumarasamy, B.Devaleenol. Factors associated with mortality among HIV-infected patients in the era of highly active antiretroviral therapy in southern India. Journal of Infectious Diseases 2010; 14:e127-e31.

[17] Andrea A., Dora L., Antonella C., Patrizia L. Simona B., et al. Prevalence, associated factors, and prognostic determinants of AIDS-Related toxoplasmic encephalitis in the era of advanced highly active antiretroviral therapy, Italy. Clinical Infectious Diseases 2004; 39:1681-91.

[18] Pate P., Bush T., Mayer K.H., Desai S., Henry K., et al. Prevalence and risk factors associated with herpes simplex virus-2 infection in a contemporary cohort of HIV-infected persons in the United States. Sex Transm Dis. 2012 Feb; 39(2):154-60.

[19] Manosuthi W., Chaovavanich A., Tansuphaswadikul S., Prasithsirikul W., Inthong Y., et al. Incidence and risk factors of major opportunistic infections after initiation of antiretroviral therapy among advanced HIV-infected patients in a resource-limited setting. Epub 2007 Nov; 55(5):464-9.

[20] Bruno L., Matthias E., V'eronique E., Rainer W., Bernard H.,et al. AIDS-related opportunistic illness occurring after initiation of potent antiretroviral therapy, the Swiss HIV cohort study. JAMA December 15, 1999; 282(23):222o-2226.

[21] Elena L., Yazdan Y., Sylvie D.B.,Bingxia W., Lindsey L., et al. the independent effect of highly active antiretroviral therapy on severe opportunistic disease incidence and mortality in HIV infected adults in Côte d'Ivoire France. AntivirTher 2007; 12(4):543-51.
[22] Matthias E., Margaret M., Geneviève C., Andrew N.P., Bruno L.et al. Prognosis of HIV-1-infected patients starting highly active antiretroviral therapy: a collaborative analysis of prospective studies. Lancet 2002; 360:119-29.

[23] CD4 cell count and the risk of AIDS or death in HIV infected adults on combination antiretroviral therapy with a suppressed viral load: a longitudinal cohort study from COHERE. PLoS Medicine March 2012; 9(3):e1001194.

[24] Antimicrobial therapy for the treatment of opportunistic infections in HIV/AIDS patients: a critical appraisal. HIV/AIDS - Research and Palliative Care 2011; 319:33.

[25] Powderly J. Clinical Evidence HIV: primary and secondary prophylaxis for opportunistic infections. Clinical Evidence 2010; 06:908.

[26] James D.C., David M., Richard D., Frank K., Willy W., et al. HIV-Infected Ugandan adults taking antiretroviral therapy with CD4 Counts .200 Cells/lLwho discontinue cotrimoxazole prophylaxis have increased risk of malaria and diarrhea. Clinical Infectious Diseases 2012; 54(8):1204-11.

[27] Wafaa M.E., Roberta L.H.,Teresa M.Y., Janice W., Donald A., et al. A randomized trial of daily and thrice-weekly trimethoprim-sulfamethoxazole for the Prevention of Pneumocystis carinii Pneumonia in Human Immunodeficiency Virus-Infected Persons. Clinica Infectious Disease 29 October 1999; 29:775-83.

[28] P.G. Sow KTMC, A.T. Dia, I. Traore. Predictors of ART adherence among HIV infected individuals in Dakar, Senegal. Journal of Medicine and Medical Science April 2012; 3(4):212-6. 